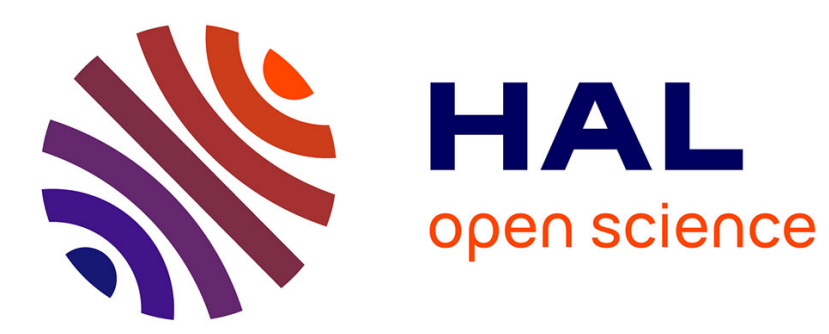

\title{
Self phase modulation of a Gaussian laser beam through a non linear thin film
}

\author{
F. Bloisi, L. Vicari, S. Martellucci, J. Quartieri, P. Cavaliere
}

\section{To cite this version:}

F. Bloisi, L. Vicari, S. Martellucci, J. Quartieri, P. Cavaliere. Self phase modulation of a Gaussian laser beam through a non linear thin film. Revue de Physique Appliquée, 1989, 24 (3), pp.411-415. 10.1051/rphysap:01989002403041100 . jpa-00246063

\section{HAL Id: jpa-00246063 https://hal.science/jpa-00246063}

Submitted on 1 Jan 1989

HAL is a multi-disciplinary open access archive for the deposit and dissemination of scientific research documents, whether they are published or not. The documents may come from teaching and research institutions in France or abroad, or from public or private research centers.
L'archive ouverte pluridisciplinaire HAL, est destinée au dépôt et à la diffusion de documents scientifiques de niveau recherche, publiés ou non, émanant des établissements d'enseignement et de recherche français ou étrangers, des laboratoires publics ou privés. 
Classification

Physics Abstracts

$42.65 \mathrm{~J}-42.10-42.30$

\title{
Self phase modulation of a Gaussian laser beam through a non linear thin film
}

\author{
F. Bloisi $\left({ }^{1}\right)$, L. Vicari $\left({ }^{1}\right)$, S. Martellucci $\left({ }^{2}\right)$, J. Quartieri $\left({ }^{2}\right)$ and P. Cavaliere $\left({ }^{3}\right)$ \\ (1) Dipartimento di Scienze Fisiche, Università di Napoli, I 80125 Napoli, Italy \\ ( $\left.{ }^{2}\right)$ Dipartimento di Ingegneria Meccanica, Università Tor Vergata, I 00100 Roma, Italy \\ $\left({ }^{3}\right)$ Istituto di Fisica, Università di Lecce, I 73100 Lecce, Italy
}

(Reçu le 29 mars 1988, révisé les 30 juin et 14 novembre 1988, accepté le 14 novembre 1988)

\begin{abstract}
Résumé. - Après quelques expériences numériques, nous avons mis au point une technique permettant l'évaluation rapide d'une intégrale apparaissant dans l'étude mathématique des anneaux de diffraction produits par un faisceau laser gaussien auto-focalisé dans une couche mince de matériau non linéaire. Nous montrons que les résultats obtenus à l'aide d'un simple micro-ordinateur (IBM-PC) peuvent être utilisés pour en déduire la valeur du coefficient de non-linéarité de la couche étudiée.
\end{abstract}

\begin{abstract}
After some numerical experience, we developed a technique allowing the fast computation of an integral appearing in the mathematical study of the diffraction rings produced by the self phase modulation of a Gaussian laser beam through a non linear thin film. The results obtained by means of a simple microcomputer (IBM-PC) are shown to may be used for the deduction of the non linearity properties of the film.
\end{abstract}

\section{Introduction.}

The focusing of a Gaussian laser beam into a non linear thin film give rise to the formation of spatial rings in the Fraunhofer diffraction pattern. This effect has been studied by several authors [1-14] since it has found several application in bistable devices, passive self limiter and non linear media characterization. Passing trough a thin film the amplitude, and hence the intensity of the beam is not modulated while the dependence of the refractive index on the intensity results in a phase modulation. In this paper we restrict our attention to Kerr or Kerr like media in which the total phase shift is proportional to the intensity. This behavior may also be taken as a valid approximation for thermal effects $[4,8,13]$. A comparison between the measured and the theoretically predicted diffraction pattern allow the computation of the non linearity coefficient. The diffraction pattern is described by the Kirchhoff integral whose computation is usually quite difficult. With an appropriate experimental apparatus the diffraction pattern may be measured in the far field and, under these hypotheses, the Kirchhoff integral may be calculated in the Fraunhofer simplification. Yet an analytical solution doesn't always exist. It has been reported in literature $[10,13]$ for a Gaussian beam but it has the form of a series quite difficult to be calculated also by means of electronic computers. Hence the theoretical study of the diffraction pattern has usually to be performed by numerical methods. The process is quite cumbersome and time consuming because the product of several oscillating functions appears in the Fraunhofer integral and hence very little integration steps have to be considered. To simplify the computation a different approach may be used. The diffraction pattern is characterized by means of some parameters, as the number and the position of the maxima, which are calculated under simplifying hypothesis and then are compared to the measured ones. In reference [6] it was observed that the number of bright rings has nearly to be the total phase shift divided by $2 \pi$ and the angular position of the outermost bright ring nearly equals the maximum of the radial derivative of the phase divided by the wave number $k$. This approximation is commonly used for the characterization of non linear media. In a recent letter [15] we introduced a new mathematical approach to the computation of circularly symmetric diffraction patterns. In this paper we apply it to the systematic study of the position of the first five maxima (bright rings) and 
minima (dark rings) in the diffraction pattern as a function of the input power, of the non-linearity coefficient and of the geometrical parameters of the experiment. To test the validity of the method we perform a comparison between our solution and the analytical one in a region were it is accessible. In the conclusions we present the position, in dimensionless units, of the first five minima and maxima of the diffraction pattern. The comparison with measured diffraction pattern will allow the straightforward calculation of the non linearity coefficient of thin films. The calculation is quick and easy as the one of reference [6] but it has an accuracy that otherwise is allowed only by the cumbersome and time consuming traditional numerical approach.

\section{Diffraction equations.}

The intensity of the incident laser beam at the entrance plane of the non linear film is

$$
I=I_{0} \exp \left(-2 r^{2} / W^{2}\right)
$$

where $W$ is the beam waist and $I_{0}$ is the on axis intensity. If the incident beam is focused on the thin film the phase is constant. The intensity distribution at the observation plane, in the Fraunhofer approximation, is

$$
\begin{aligned}
& I(r, z)=\left(k^{2} I_{0} / z^{2}\right) \mid \int_{0}^{\infty} \mathrm{d} r_{1} r_{1} J_{0}\left(k r_{1} r / z\right) \times \\
& \times\left.\exp \left(-r_{1}^{2} / W^{2}\right) \exp \left[-i 2 \pi \Phi_{0} \exp \left(-2 r_{1}^{2} / W^{2}\right)\right]\right|^{2}
\end{aligned}
$$

where $k$ is the wave number, $2 \pi \Phi_{0}$ is the total phase shift at intensity $I_{0}, J_{0}$ is the first kind Bessel function of order zero.

Equation (2) may be more conveniently written

$$
\begin{aligned}
E(y)= & \mid \int_{0}^{\infty} \mathrm{d} x x J_{0}(y x) \times \\
& \times\left.\exp \left[-x^{2}-i 2 \pi \Phi_{0} \exp \left(-2 x^{2}\right)\right]\right|^{2}
\end{aligned}
$$

where $E=I z^{2} / I_{0} k^{2} W^{4}, \quad x=r_{1} / W, \quad y=k r W / z$. Equation (3) has the advantage that $E, x$ and $y$ are dimensionless quantities.

\section{Fraunhofer diffraction pattern.}

The normalized intensity distribution $E(y)$ given by equation (3) may be expressed as a series

$$
E(y)=\left|\sum_{s=0}^{\infty} \frac{\left(-i 2 \pi \Phi_{0}\right)^{s}}{s !} \frac{\exp \left[-y^{2} /(8 s+4)\right]}{4 s+2}\right|^{2}
$$

but some difficulties may be found for a numerical evaluation of $E(y)$ as given by equation (4). Obviously the summation has to be truncated somewhere.
The real part of the series is given by the even values of $s$ while the imaginary part is given by the odd values of $s$. Both these parts are made of alternating terms whose relative importance is function of both $y$ and $s$. For large values of $y$ terms with large values of $s$ are more important than terms with low ones. Hence the summation has to be performed for several thousands of terms. Owing to these difficulties direct numerical integration of equation (3) is usually preferred [12].

A more convenient expression for $E$, requiring the summation of decreasing real terms can be obtained with the assumptions that both source and output intensity distributions are zero outside of a finite support. Although it is well known that a function and its Hankel transform cannot be both limited to a finite support, these assumptions are often "physically true. A good example is given by the gaussian beam we are dealing with. The Hankel transform of a Gaussian function is a Gaussian. Of course from a mathematical point of view the Gaussian function extend to the infinity. Nevertheless from a physical point of view it may be disregarded when the radius is about five times larger than the beam waist and the amplitude is reduced by a factor $\exp (-25)$. It has been reported [6] that the diffraction pattern of a gaussian beam with Gaussian phase modulation significantly differs from zero only when the angle of view is lesser than the maximum of the radial derivative of the phase divided by the wave number $k$. The method we are using cannot be applied to plane waves or to any wave whose energy isn't finite but may be successfully used for almost all actual beam shapes with finite energy (i.e. circle function even of very large radius, etc.). Further discussion of these hypotheses is performed in the following when they are used.

The normalized diffraction intensity distribution given by equation (3) may be written as

$$
E(y)=|F(y)|^{2}
$$

where

$$
F(y)=H\{f(x)\}=\int_{0}^{\infty} x \mathrm{~d} x J_{0}(y x) f(x)
$$

is the Hankel transform of the normalized source amplitude distribution

$$
f(x)=\exp \left[-x^{2}-i 2 \pi \Phi_{0} \exp \left(-2 x^{2}\right)\right] .
$$

For the inversion property of the Hankel transform it holds

$$
f(x)=H\{F(y)\}=\int_{0}^{\infty} y \mathrm{~d} y J_{0}(y x) F(y) .
$$

We know that the energy has to be finite, hence the integral

$$
\int_{0}^{\infty} y \mathrm{~d} y F(y)
$$


has to be finite and hence the function $F(y)$ has to go to decrease faster than the inverse of the square radius. Since in the integral (7) $F(y)$ is multiplied by $J_{0}(y x)$ whose absolute value decreases with the inverse of the radius the integral

$$
\int_{y^{*}}^{\infty} y \mathrm{~d} y J_{0}(y x) F(y)
$$

decreases at least with the inverse of $y$. Once given an $y^{*}$ so that this integral may be disregarded it may be assumed that $F(y)=0$ for $y>y^{*}$. In fact $F(y)$ decreases faster than this integral. Now the integral (7) reduces to

$$
f(x)=H\{F(y)\}=\int_{0}^{y^{*}} y \mathrm{~d} y J_{0}(y x) F(y) .
$$

The function $F(y)$ is a continue function for physical reasons and hence it satisfies the Dirichlet conditions in the closed interval $0 \leqslant y \leqslant y^{*}$. When a function has these properties it may be developed in terms of Bessel functions by means of the finite Hankel transform [16]. If we select the basis given by the zeroth order Bessel functions it assumes the simple form

$$
F(y)=\frac{2}{y^{* 2}} \sum_{s=1}^{\infty} \frac{J_{0}\left(j_{s} y / y^{*}\right)}{\left[J_{1}\left(j_{s}\right)\right]^{2}} F_{s}
$$

where $J_{1}$ is the first kind Bessel function of order one, $j_{s}$ is the $s$-th positive zero of $J_{0}$ and $F_{s}$ is defined by

$$
F_{s}=\int_{0}^{y^{*}} y \mathrm{~d} y J_{0}\left(y j_{s} / y^{*}\right) F(y) .
$$

Comparison with equation (7) shows that

$$
F_{s}=f\left(j_{s} / y^{*}\right)
$$

and hence

$$
F(y)=\frac{2}{y^{* 2}} \sum_{s=1}^{\infty} \frac{J_{0}\left(j_{s} y / y^{*}\right)}{\left[J_{1}\left(j_{s}\right)\right]^{2}} f\left(j_{s} / y^{*}\right) .
$$

Since the energy has to be finite at the input plane as well at the output one, the same considerations developed for $F(y)$ hold for $f(x)$ that has to decrease at least as the inverse square of the radius. In our case they have a Gaussian decay and hence the terms of the series in equation (9) quickly decreases with increasing $s$. Hence it may be found an integer $N$ so that it may be assumed $f\left(j_{s} / y^{*}\right)=0$ for $s \geqslant N$. Once again this condition may be mathematically satisfied if $f$ vanishes out of a disk. Elsewhere it may be chosen so that $f$ is so small that it may be physically disregarded. Hence

$$
\begin{aligned}
E & =\mid \frac{2}{y^{* 2}} \sum_{s=1}^{N} \frac{J_{0}\left(j_{s} y / y^{*}\right)}{\left[J_{1}\left(j_{s}\right)\right]^{2}} \times \\
& \times\left.\exp \left[-\frac{j_{s}^{2}}{y^{* 2}}-i 2 \pi \Phi_{0} \exp \left(-\frac{j_{s}^{2}}{y^{* 2}}\right)\right]\right|^{2} .
\end{aligned}
$$

In the following we assumed $N=200$, a value that appears to be sufficient in many cases of physical interest. In figure 1 we show a diffraction pattern computed by means of equation (10) (full line) and of equation (4) (crosses). We encountered severe difficulties to handle the numbers required by equation (4) with our computer (IBM-PC) for higher values of $\Phi_{0}$. Of course direct numerical integration of equation (2) could be used but to achieve an accuracy comparable to the one used in this paper it would require computer times of two order of magnitude higher.

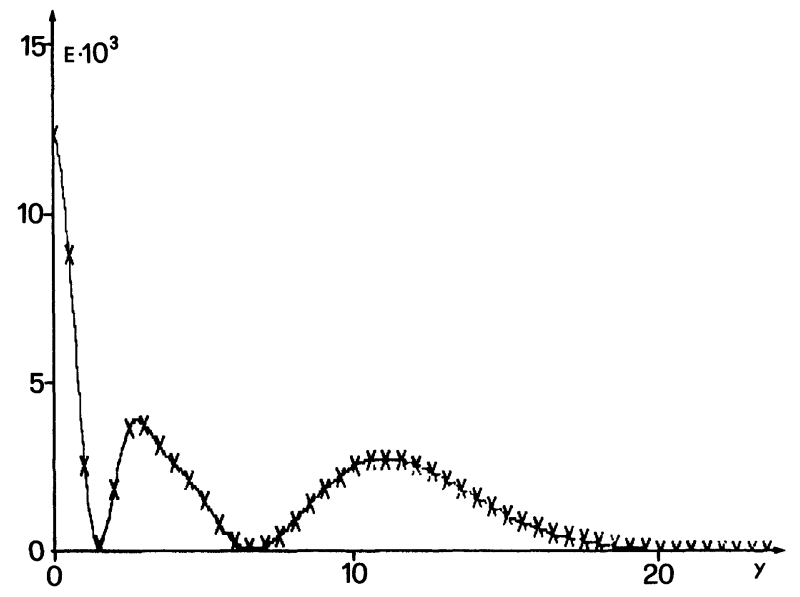

Fig. 1. - Diffraction pattern for $\Phi_{0}=2.0$ obtained with our method (full line) and using analytic solution (crosses).

\section{Conclusions.}

Computer implementation of equation (10) was used to compute 500 diffraction patterns for $0 \leqslant \Phi_{0} \leqslant 5$. Each diffraction pattern was composed by 200 values of $E$ given by equation (10). The position of the maxima of the diffraction patterns are reported in figure 2 while the position of minima are reported in figure 3 . For a discussion let us see figure 4 where full lines represent the maxima positions, dashed lines represent the minima position and crosses represent positions in which there is a point of inflection due to the merging of a maximum and a minimum. The dash dotted line through zero is the theoretical position of the external maximum in the approximation of reference [6]. Several couples of maxima and minima arise and evolve as intensity increases. The first couple $\left(\mathrm{A}^{+}, \mathrm{A}^{-}\right)$arise for $\Phi_{0}=0.55$ at $y=2.3$ and stay around the optical axis $y=0$. The other couples of maxima and minima $\left(\mathrm{B}^{+}, \mathrm{B}^{-}\right),\left(\mathrm{C}^{+}, \mathrm{C}^{-}\right),\left(\mathrm{D}^{+}, \mathrm{D}^{-}\right),\left(\mathrm{E}^{+}, \mathrm{E}^{-}\right)$arise in the range $3.8 \leqslant y \leqslant 3.9$ for several values of $\Phi_{0}$. After an initial assessment their distances from the optical axis increase linearly with the intensity. For 


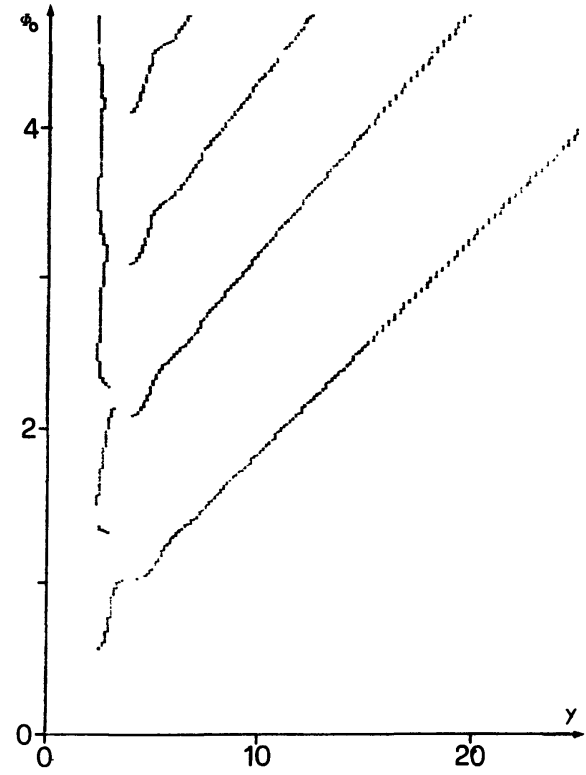

Fig. 2. - The position $(y=k r W / z)$ of the maxima (bright rings) of the diffraction patterns are shown for 500 values of $\Phi_{0}$ in the range $[0,5]$.

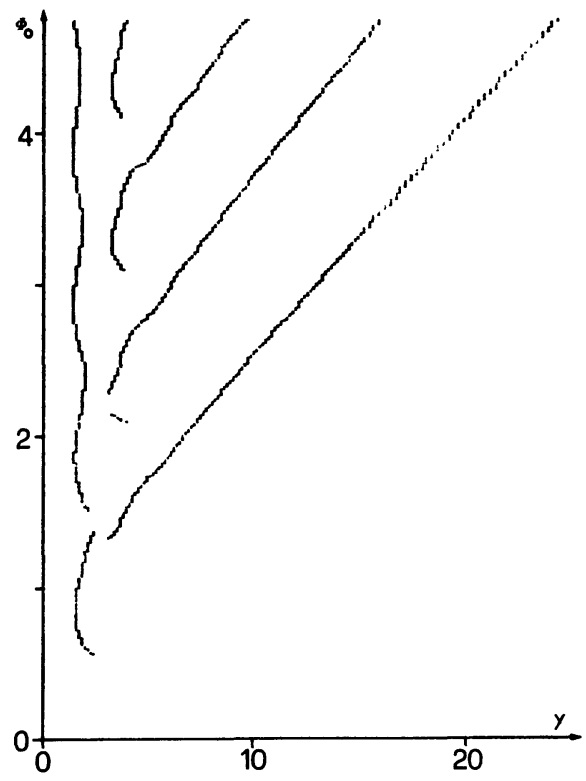

Fig. 3. - The position $(y=k r W / z)$ of the minima (dark rings) of the diffraction patterns are shown for 500 values of $\Phi_{0}$ in the range $[0,5]$.

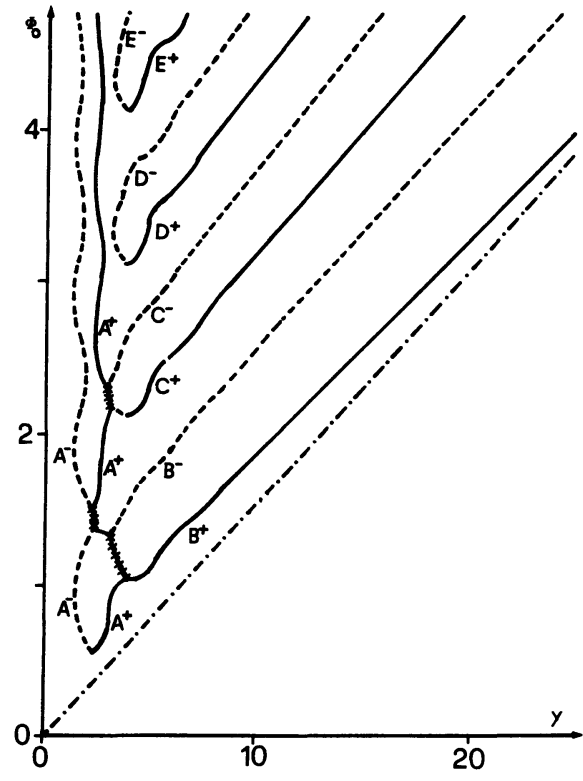

Fig. 4. - The position $(y=k r W / z)$ of the maxima (full line) and of the minima (dashed line) of the diffraction patterns are shown for values of $\Phi_{0}$ in the range $[0,5]$. The crosses represents the positions of flexes due to the merging of the maximum $\mathrm{A}^{+}$with the minima $\mathrm{B}^{-}$, $\mathrm{A}^{-}$or $\mathrm{C}^{-}$. The dash-dot line is the position of the outermost bright ring estimated following reference [6] : $y=(\sqrt{8} \pi / e) \Phi_{0}$.

$\Phi_{0}>1.5$ the external maximum $\mathrm{B}^{+}$follows the straight line $y=7.0858 \Phi_{0}-3.179$ instead of the straight line $y=6.5378 \Phi_{0}$ provided by reference [6]. We observe that in some regions the maximum $\mathrm{A}^{+}$disappear since it merges to the minima $\mathrm{B}^{-}, \mathrm{A}^{-}$or $\mathrm{C}^{-}$giving rise to an inflection point. The number and the position of the maxima and minima are very well defined for $\Phi_{0}=2, \Phi_{0}=3$ or $\Phi_{0}=4$. These values hence have to be preferred in experimental works. The maxima position may be marked on the screen and the intensity of the beam may be increased until the actual maxima coincide with the marked ones. The nonlinearity coefficient may hence be easily computed from the intensity value.

\section{References}

[1] Akhmanov S. A., Khokhlov R. V., Sukhorukov A. P., Laser Handbook, Eds. F. T. Arecchi, E. O. Schulz-Dubois (North-Holland, Amsterdam) 1972, vol. 2 part E3.

[2] SHEN Y. R., The Principles of Nonlinear Optics (John Wiley \& Sons, New York) 1984, Chap. 17.
[3] Akhmanov S. A., Krindach D. P., Migulin A. V., SuKHORUKOV A. P., KHOKHLOV R. V., IEEE J. Quantum Electron. QE-4 (1968) 568.

[4] Dabby F. V., Gustafson T. K., Whinnery J. R., Kohanzadeh Y., Kelley P. L., Appl. Phys. Lett. 16 (1970) 362.

[5] Zolot'ko A. S., Kitaeva V. F., Kroo N., 
Sobolev N. N., Chillag L., JetP Lett. 32 [11] Hsiung H., Shi L. P., Shen Y. R., Phys. Rev. A 30 (1980) 158. (1984) 1453.

[6] Durbin S. D., Arakelian S. M., Shen Y. R., Opt. [12] Santamato E., Shen Y. R., Opt. Lett. 9 (1984) 564. Lett. 6 (1981) 411.

[13] Slaby J., Opt. Commun. 60 (1986) 133.

[7] BJoRKholm J. E., SMith P. W., TOMLinson W. J., KaPlan A. E., Opt. Lett. 6 (1981) 345.

[8] Kноо I. C., Phys. Rev. A 25 (1982) 1636.

[14] KHOO I. C., Hou J. Y., LiU T. H., YaN P. Y., Michael R. R., Finn G. M., J. Opt. Soc. Am. B 4 (1987) 886.

[9] Khoo I. C., Finn G. M., Michael R. R., Liu T. H., [15] Bloisi F., Martellucci S., Quartieri J., Vicari Opt. Lett. 11 (1986) 227.

[10] KhOo I. C., Yan P. Y., LiU T. H., ShePaR S., HoU J. Y., Phys. Rev. A 29 (1984) 2756. L., Europhys. Lett. 4 (1987) 905.

[16] SNEDdON I. N., Fourier Transforms (McGraw-Hill, New York, Toronto, London) 1951. 